\title{
Ventilation-Induced Massive Lethal Air Embolism and Subcutaneous Emphysema in a Patient With a Lung Cavern
}

\author{
Wim Verelst MD, Walter Verbrugghe MD, Martin Lammens MD PhD, \\ Annemie Snoeckx MD, and Philippe G Jorens MD PhD
}

\begin{abstract}
The simultaneous occurrence of subcutaneous emphysema and intravascular air due to an air embolism is a rare condition. Here, we report a patient with COPD who developed a severe episode of hemoptysis due to rupture of a previously undiagnosed lung cavern. Intubation and ventilation led to the development of both massive subcutaneous emphysema and a massive air embolism, resulting from aspiration of air through a torn pulmonary vessel in the cavern. The dramatic amount of intravenous air and subsequent conduction along the venous system to the right heart and pulmonary trunk caused major hemodynamic compromise and ultimately death. The degree of subcutaneous emphysema, especially the massive venous air embolism, was unprecedented. Key words: hemoptysis; lung cavern; subcutaneous emphysema; air embolism; fatality; computed tomography scan. [Respir Care 2015;60(1):e6-e10. @ 2015 Daedalus Enterprises]
\end{abstract}

\section{Introduction}

Barotrauma is physical damage to body tissues caused by a difference in pressure between a gas space inside the body or in contact with the body and the surrounding space. Pulmonary barotrauma usually refers to alveolar rupture due to elevated transalveolar pressure allowing air from the alveolus to enter the pulmonary interstitium. ${ }^{1}$ The interstitial air can then dissect along the perivascular sheaths toward the mediastinum, leading to a pneumothorax, pneumomediastinum, or subcutaneous emphysema when air is trapped in the mediastinum and the subcutaneous layer of skin of the chest, neck, and face. A bronchopleural fistula, tension pneumothorax, and air embolism are the most severe, albeit infrequent, complications of barotraumas. ${ }^{1}$

\footnotetext{
Drs Verelst, Verbrugghe, and Jorens are affiliated with the Department of Critical Care Medicine; Dr Lammens is affiliated with the Department of Pathology; and Dr Snoeckx is affiliated with the Department of Radiology, Antwerp University Hospital, University of Antwerp, Edegem, Belgium.
}

The authors have disclosed no conflicts of interest.

Correspondence: Philippe G Jorens MD PhD, Department of Critical Care Medicine, Antwerp University Hospital (UZA), Wilrijkstraat 10, 2650 Edegem, Belgium. E-mail: philippe.jorens@uza.be.

DOI: $10.4187 /$ respcare.03194
Positive-pressure ventilation (the most common type of mechanical ventilation) places patients at risk for pulmonary barotrauma. Here, we report a lethal case of pulmonary barotrauma resulting in both massive subcutaneous emphysema and an air embolism, which occurred after intubation of a patient with a lung cavern immediately following initiation of positive-pressure ventilation.

\section{Case Report}

A 68-y-old female presented to the emergency department with a 2-week history of productive cough, generalized arthritis, and fever. She had a medical history of rheumatoid arthritis, COPD, and obesity and was treated with infliximab, methotrexate, omeprazole, and low-dose methylprednisolone.

At presentation, the patient was pale and tachypneic (breathing frequency of 22 breaths $/ \mathrm{min}$ ) and had a sinus tachycardia (120 beats/min) but normal blood pressure $(128 / 58 \mathrm{~mm} \mathrm{Hg})$. The peripheral transcutaneous pulse oximetry measurement was $96 \%$, and the tympanic body temperature was $38.2^{\circ} \mathrm{C}$. Chest auscultation demonstrated bilateral crackles, which were more distinct on the right side. The remainder of the physical examination was unremarkable.

Blood results showed no profound anemia, with a hematocrit of $31 \%$ and hemoglobin at $10.4 \mathrm{~g} / \mathrm{dL}$. The patient had a white blood cell count of 12,000 cells/ $\mu \mathrm{L}$ (normal 


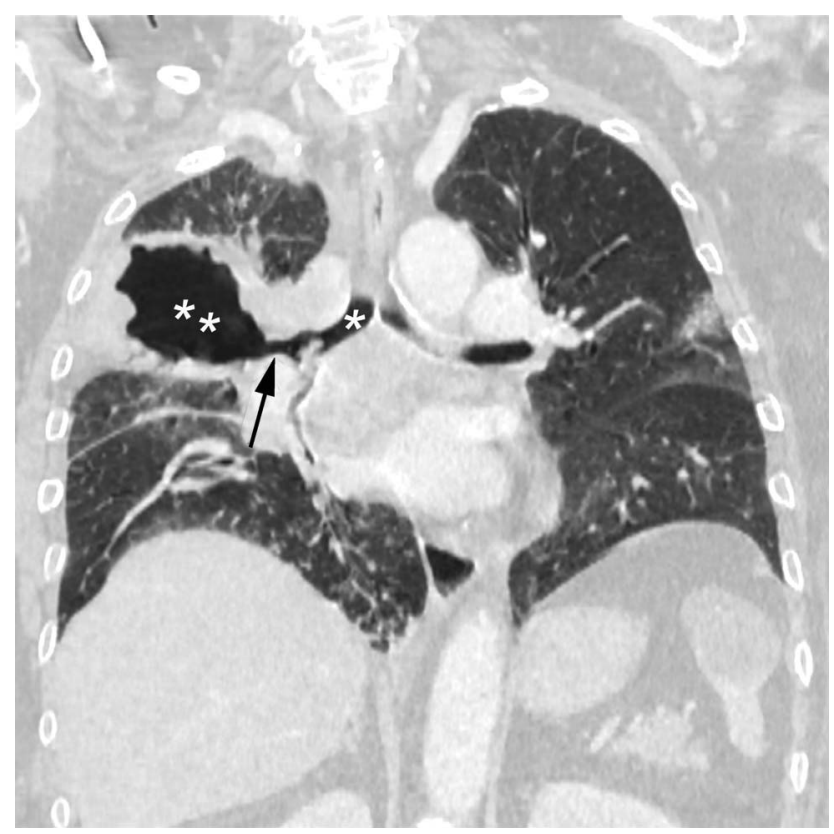

Fig. 1. Coronal computed tomography image (lung window setting) confirmed a large ill-defined mass with central cavitation (double asterisk). The lesion had an irregular wall with variable thickness. There was continuity between the air in the lesion and the air in the right main bronchus (single asterisk). This observation implicates a fistula between both structures, which is well depicted (arrow).

range is $4,000-10,500$ cells $/ \mu \mathrm{L}$ ) and an elevated Creactive protein level of $23 \mathrm{mg} / \mathrm{dL}$ (normal values are $<0.5 \mathrm{mg} / \mathrm{dL}$ ). Blood chemistry, including serum electrolytes and creatinine, was normal. Arterial blood gas values, without administration of supplemental oxygen, revealed a $\mathrm{pH}$ of $7.56, \mathrm{P}_{\mathrm{CO}_{2}}$ of $31.4 \mathrm{~mm} \mathrm{Hg}, \mathrm{HCO}_{3}{ }^{-}$at 28 $\mathrm{mmol} / \mathrm{L}$, and $\mathrm{P}_{\mathrm{aO}_{2}}$ of $80.8 \mathrm{~mm} \mathrm{Hg}$ with a corresponding arterial oxygen saturation of $95 \%$.

Unexpectedly, a chest radiograph obtained in the emergency department showed a giant lung cavern in the right upper lobe that was suggestive of a necrotizing tumor or abscess (not shown). A computed tomography (CT) scan was performed the same day for further diagnosis and showed a thick-walled $8 \times 10 \mathrm{~cm}$ cavern with a necrotic aspect connected to the right main bronchus (Fig. 1).

Immediately following the $\mathrm{CT}$ procedure, the patient developed massive hemoptysis in the radiology ward, with a drop in arterial oxygen saturation and decreased responsiveness. The resuscitation team arrived minutes later, and the patient was intubated and ventilated by a board-certified and experienced ICU physician in a single uneventful attempt. Positive-pressure ventilation (pressure controlled ventilation included inspiratory pressure of $24 \mathrm{~mm} \mathrm{Hg}$, tidal volumes of $500 \mathrm{~mL}$, breathing frequency of 12 breaths $/ \mathrm{min}$, and PEEP of $5 \mathrm{~mm} \mathrm{Hg}$ ) was initiated. The patient subse- quently and immediately developed massive subcutaneous emphysema. After hemodynamic stabilization (a systolic blood pressure above $100 \mathrm{~mm} \mathrm{Hg}$ with a sinus tachycardia of 110 beats $/ \mathrm{min}$ ), she was stable enough to be put through the scanner and was rescanned immediately.

The second CT scan showed a large quantity of blood in the cavern and the tracheobronchial tree. Moreover, there was a major quantity of air in the complete pulmonary and systemic venous system, the right heart, and the pulmonary arteries (Fig. 2). Most likely, this massive amount of intravascular air was caused by air entry in a ruptured pulmonary vessel in the cavern following positive-pressure ventilation. Pulmonary rupture through the cavern had also caused a right-sided secondary pneumothorax.

Immediately following completion of the scan and despite initial stabilization, cardiac arrest ensued. Even with prolonged cardiopulmonary resuscitation, the patient died. After obtaining consent from the family, an autopsy was performed.

Macroscopically, the principal finding was extensive facial, cervical, and thoracic subcutaneous emphysema. There was also pronounced abdominal distention due to the presence of a large amount of intraperitoneal air. After opening the abdomen, air escaped, and there was crepitation of the peritoneal fat as well as signs of air trapping (via the periesophageal space and other breaches).

The right lung showed a pleural defect between the middle and inferior lobes, with bulging necrotic hemorrhagic tissue that formed a breach between the cavity and the thoracic space. The mechanism of air entry in the peritoneum is a consequence of the mechanical ventilation-induced pneumothorax: intrathoracic pressure buildup enabled air to escape from the thoracic cavity by passing through small diaphragmatic fenestrations, thereby entering the peritoneal space.

A mass $(6.5 \times 5.5 \mathrm{~cm})$ was found wrapped around the right main bronchus with a direct connection to the lung cavern $(10 \times 8.5 \mathrm{~cm})$ in the upper and middle lobes (Fig. 3 ). Filled with hemorrhagic and necrotic material, this cavern drained into the defect between the middle and inferior lobes. Multiple enlarged lymph nodes were found in the proximity of the hilar region. The right heart and pulmonary trunk were filled with foamy red blood, which we had anticipated considering the $\mathrm{CT}$ findings.

On microscopy, the mass consisted of a poorly differentiated non-small cell lung carcinoma. Tuberculosis was not retained as the cause of the cavity, as culture of both gastric and respiratory samples did not reveal mycobacteria, and Ziehl-Neelsen staining did not show acid-fast organisms. On autopsy, there were no granulomas suggestive of tuberculosis. Interstitial air was found in the lung tissue. More importantly, multiple recent emboli were disseminated in small vessels. These emboli consisted mostly of fibrin, with some nuclear debris and polymorphonuclear 


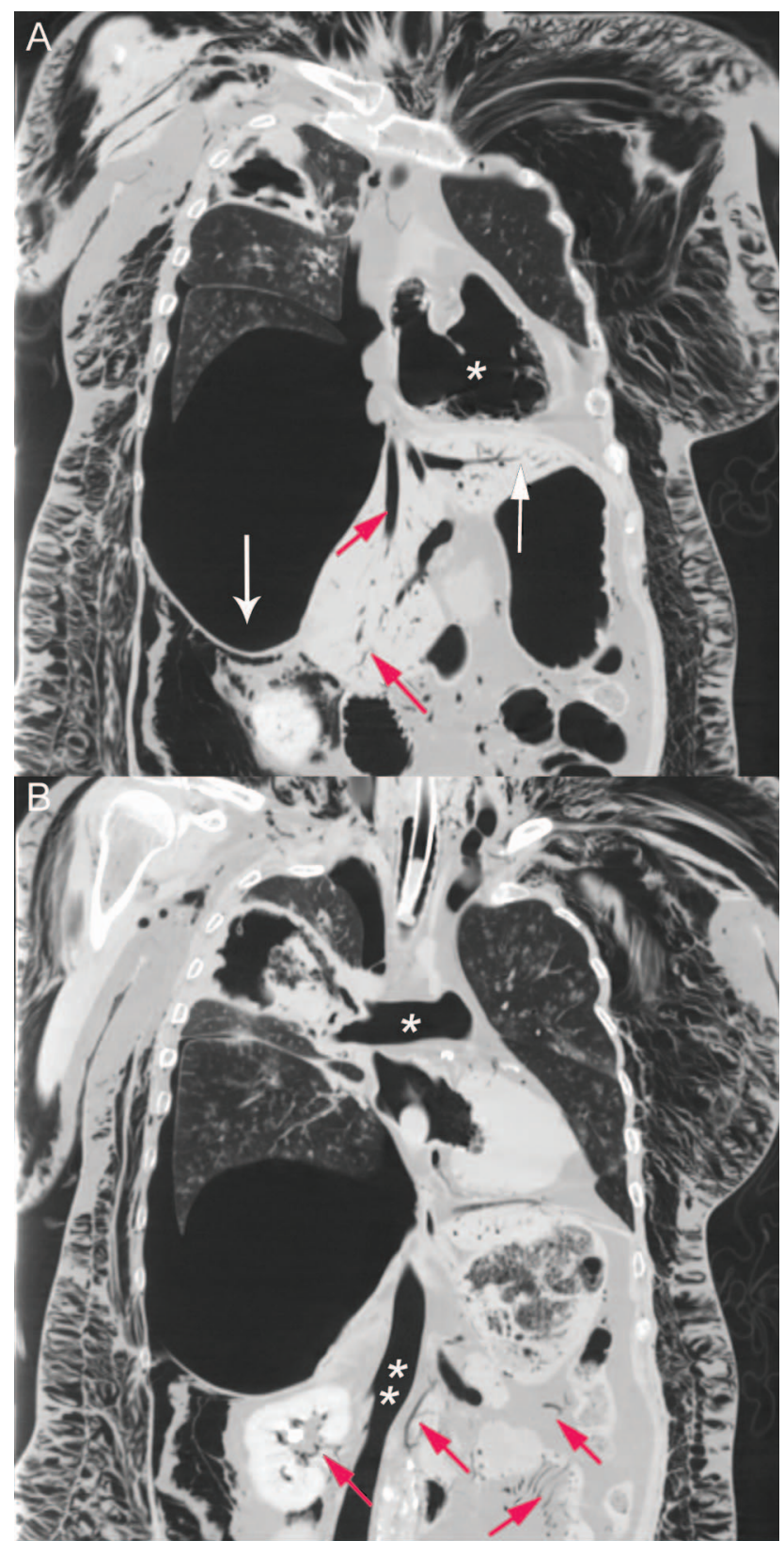

Fig. 2. A: Computed tomography examination (lung window setting) after intubation and during resuscitation. A coronal image at the level of the heart shows marked thickening of the subcutaneous fat with a massive amount of subcutaneous emphysema. There is downward displacement of the right diaphragm (white arrow) with a large secondary pneumothorax. The normal density of the venous (and partially arterial) system is extensively replaced by air. The right side of the heart (asterisk) is distended and completely filled with air. Also note air in the hepatic veins and portal system (red arrows). B: A coronal view more anterior than in A demonstrates air in the pulmonary arteries (single asterisk), the inferior vena cava (double asterisk), and small mesenteric vessels (red arrows).

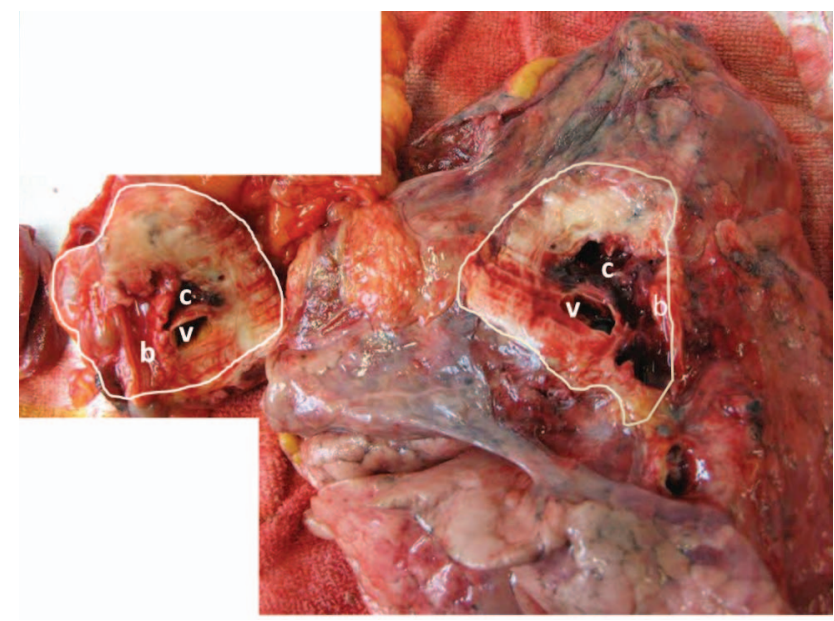

Fig. 3. Macroscopy of the right upper lobe of the lung at obduction. White contours are drawn around complementary mirror slides through a white-yellow tumoral mass with a hemorrhagic cavern (c) in continuity with the bronchus (b). The visible vessel (v) is most likely the origin of the hemorrhage.

cells that were sometimes intermingled with small air bubbles (Fig. 4).

\section{Discussion}

In the patient presented, the development of post-intubation subcutaneous emphysema was recognized as an alarming clinical sign indicating a serious complication. When subcutaneous emphysema occurs following endotracheal intubation and the initiation of positive-pressure ventilation, one should consider potential causes (eg, perforation of the hypopharynx or esophagus, tracheobronchial laceration, or lung injury [most frequently as a result of ventilation-induced barotrauma]). As the former two causes seemed rather improbable, the patient was rescanned immediately to differentiate between the latter two causes.

Although tracheobronchial rupture clearly was not the causative mechanism in this case, the patient did have some typical risk factors for intubation-related injury (ie, female gender, short stature, COPD, advanced age, and corticosteroid use). ${ }^{2-5}$ Tracheobronchial laceration is a known rare complication of endotracheal intubation, occurring more frequently with multiple intubation attempts, emergency intubation, and airway instrumentation (eg, use of a stylet)..$^{2-5}$

In this patient, vigorous coughing (most likely a symptom of pulmonary superimposed infection) led to the buildup of a pressure gradient between the alveoli and the surrounding tissues. Upon reaching a critical level, the generated pressure caused alveolar and venous rupture (resulting in bleeding) in a pulmonary weak spot (ie, the cavern). COPD and steroid use were most likely facilitat- 


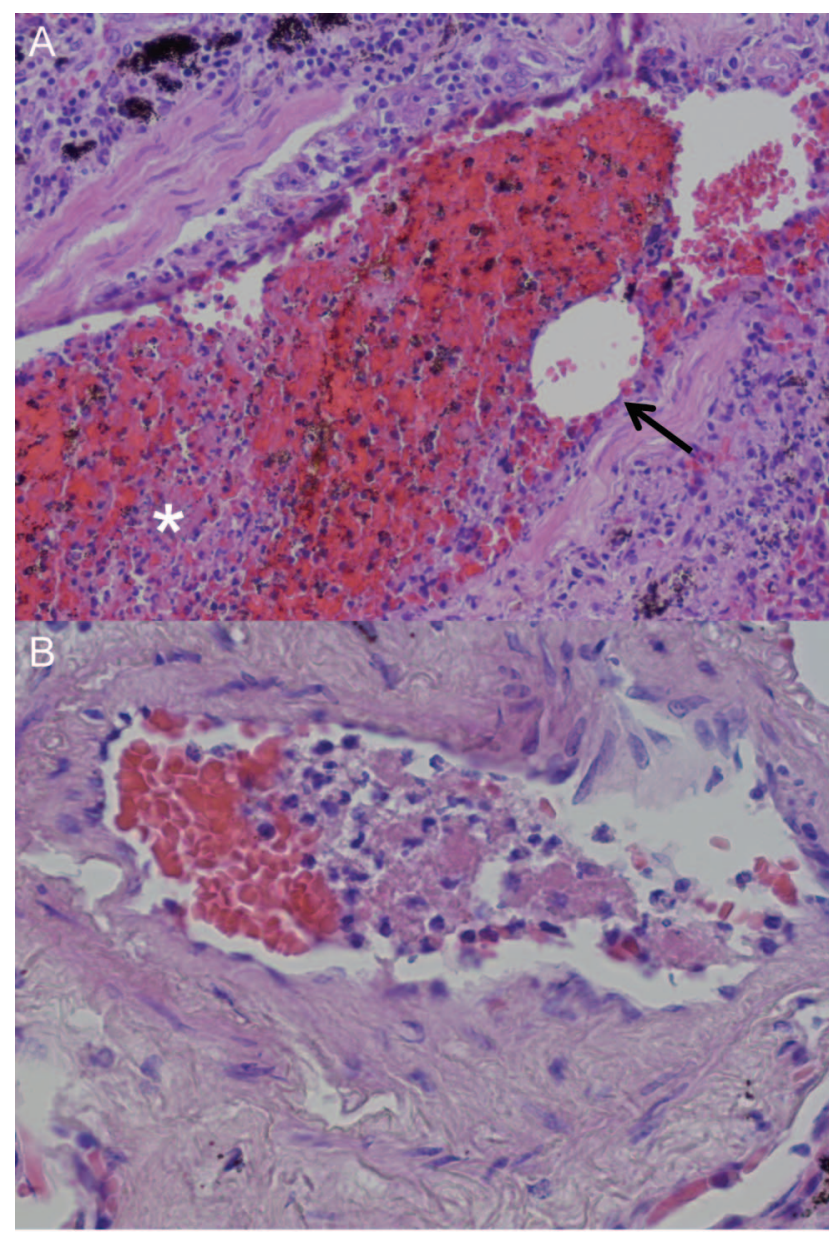

Fig. 4. Microscopy and pathology. A: Sludging in a lung vessel due to fibrin (pink-stained material marked with an asterisk), cellular debris, polymorphonuclear cells, and an air bubble (arrow). B: A lung vessel is seen, partly thrombosed by a recent embolus composed of fibrinous (pink) material with cellular debris.

ing factors in this pathophysiological process. When considering the exposure of the critical lung area to positivepressure expansion, it is very likely that positive-pressure mechanical ventilation aggravated the primary laceration.

Initiation of mechanical ventilation caused pressurized air to enter the pulmonary interstitium, creating subcutaneous emphysema through further dissection along the peribronchovascular sheath. Subcutaneous emphysema is usually benign. In our patient, primary or secondary rupture of the pleura generated a right-sided secondary pneumothorax. Such a massive amount of subcutaneous emphysema, involving the deeper tissues of the mediastinum, chest, and abdominal wall, can be life-threatening. This compression can then result in air-flow obstruction, decreased cardiac venous return, and a perfusion deficit in the head and neck region. Rigidity of the chest wall, secondary to subcutaneous air, restricts full pulmonary expansion and will lead to augmented airway pressures. ${ }^{5}$
Although the aforementioned complications alone can be life-threatening, a massive air embolism was the main process that resulted in cardiac arrest. Rupture of the cavern led to damage of a pulmonary vein, causing massive hemoptysis. Positive-pressure ventilation resulted in venous air entry and subsequently a dramatic air embolism, as illustrated by the CT and pathological findings.

Iatrogenic venous air embolisms are well-known problems, often resulting from mismanagement of a central or peripheral infusion line, and are a reported complication of percutaneous coronary intervention, laparoscopy, thoracotomy, endoscopy, arthroscopy, prostatectomy, airway laser surgery, hemodialysis, and pulmonary barotrauma. ${ }^{6,7}$ When a small amount of air of venous origin enters the pulmonary circulation, pulmonary vessel filtration prevents further embolization of air in the systemic or coronary circulation. ${ }^{6-8}$ Immediately following venous embolization, the obstruction of blood flow in the pulmonary capillary distal and proximal to the occluding gas particles causes tissue ischemia and pressure changes in the circulation and interstitium around the affected blood vessel. Treated as a foreign body, the bubble initiates an inflammatory response and complement activation. ${ }^{9}$ A local destructive process takes place, in which neutrophils play a critical role. By production of superoxide and hydroxyl radicals and release of proteolytic enzymes, which increase membrane permeability, they facilitate the generation of interstitial lung edema. In addition, by acting as a foreign substance, the air bubble activates coagulation and induces platelet aggregation, further enhancing local ischemia by plugging blood vessels. ${ }^{9}$ These mechanisms were reflected in the microscopic findings in the patient (ie, apart from intravascular air and interstitial emphysema, multiple small emboli with fibrin deposits and debris were found).

As a consequence of the previously mentioned processes, air bubble trapping in the pulmonary capillaries causes an increase in pulmonary artery pressure. The resultant resistance to right ventricular outflow will then further diminish venous return, leading to a fall in cardiac output. When a large air volume is injected abruptly into human veins, acute cor pulmonale, asystole, or both are likely to occur. ${ }^{10}$ In humans, a lethal volume of air is reported to be between 100 and $500 \mathrm{~mL}$, although much lower volumes have been reported to have caused death. ${ }^{11}$

When large quantities of air overwhelm the barrier of the pulmonary capillaries, it is possible for gas bubbles to enter the arterial and coronary circulation, causing endartery obstruction and consequently ischemia. This mechanism was demonstrated in animals as well as in humans. ${ }^{12-14}$ Considering the large volume of air entering the venous system, the occurrence of cardiac arrest in this patient was most likely attributable to acute cor pulmonale and possibly an arterial/coronary air embolism by flooding 


\section{Barotrauma Leading to Air Embolism and Subcutaneous Emphysema}

of the pulmonary air filter and not by the subcutaneous emphysema alone.

Regarding the course of this patient's history, we conclude that the development of hemoptysis set in motion a cascade of events that led to a fatal conclusion. In the acute situation, selective intubation of the left main bronchus and single ventilation of the left lung (eg, by use of a double-lumen endobronchial tube) could have perhaps halted further deterioration. However, considering that this type of equipment is generally unavailable in a resuscitation setting and recognizing that the intensive care physician (summoned in acute circumstances) was unaware of the case specifics, it is fair to state that this patient's outcome was inevitable. To our knowledge, such massive subcutaneous emphysema and an air embolism are unprecedented.

\section{REFERENCES}

1. Hyzy RC. Pulmonary barotrauma during mechanical ventilation. UpToDate. http://www.uptodate.com/contents/pulmonary-barotraumaduring-mechanical-ventilation. Accessed August 4, 2014.

2. Hashem B, Smith JK, Davis WB. A 63-year old woman with subcutaneous emphysema following endotracheal intubation. Chest 2005; 128(1):434-438.
3. Hofmann HS, Rettig G, Radke J, Neef H, Silber RE. Iatrogenic ruptures of the tracheobronchial tree. Eur J Cardiothorac Surg 2002; 21(4):649-652.

4. Schneider T, Storz K, Dienemann H, Hoffmann H. Management of iatrogenic tracheobronchial injuries: a retrospective analysis of 29 cases. Ann Thorac Surg 2007;83(6):1960-1964.

5. Gries CJ, Pierson DJ. Tracheal rupture in life-threatening subcutaneous emphysema. Respir Care 2007;52(2):191-195.

6. Muth CM, Shank ES. Gas embolism. N Engl J Med 2000;342(7): 476-482.

7. Jorens PG, Van Marck E, Snoeckx A, Parizel PM. Nonthrombotic pulmonary embolism. Eur Respir J 2009;34(2):452-474.

8. Butler BD, Hills BA. The lung as a filter for microbubbles. J Appl Physiol 1979;47(3):537-543.

9. Barak M, Katz Y. Microbubbles: pathophysiology and clinical implications. Chest 2005;128(4):2918-2932.

10. Palmon SC, Moore LE, Lundberg J, Toung T. Venous air embolism: a review. J Clin Anesth 1997;9(3):251-257.

11. Orebaugh SL. Venous air embolism: clinical and experimental considerations. Crit Care Med 1992;20(8):1169-1177.

12. Butler BD, Hills BA. Transpulmonary passage of venous air emboli. J Appl Physiol 1985;59(2):543-547.

13. Vik A, Brubakk AO, Hennessy TR, Jenssen BM, Ekker M, Slørdahl SA. Venous air embolism in swine: transport of gas bubbles through the pulmonary circulation. J Appl Physiol 1990;69(1):237-244.

14. Tommasino C, Rizzardi R, Beretta L, Venturino M, Piccoli S. Cerebral ischemia after venous air embolism in the absence of intracardiac defects. J Neurosurg Anesthesiol 1996;8(1):30-34. 